\title{
On a Computational Algorithm to the HJE in Nnlinear $\mathrm{H} \infty$ Control
}

Shr-Shiung $\mathrm{Hu}$

Engineering Officer, The 202nd Arsenal, C.S.F., P. O. Box 90582-22, Nankang, Taipei 115, Taiwan, R.O.C.

Pao-Hwa Yang

Assistant Professor, Department of Mechanical Engineering, Hsiuping Institute of Technology, Dah-Li City, Taichung County 412, Taiwan, R.O.C.

Jeng-Yih Juang

Professor, Department of Merchant Marine, National Taiwan Ocean University, Keelung 202, Taiwan, R.O.C.

Follow this and additional works at: https://jmstt.ntou.edu.tw/journal

Part of the Mechanical Engineering Commons

\section{Recommended Citation}

$\mathrm{Hu}$, Shr-Shiung; Yang, Pao-Hwa; and Juang, Jeng-Yih (2001) "On a Computational Algorithm to the HJE in Nnlinear H $\infty$ Control," Journal of Marine Science and Technology. Vol. 9: Iss. 2, Article 4.

DOI: 10.51400/2709-6998.2439

Available at: https://jmstt.ntou.edu.tw/journal/vol9/iss2/4

This Research Article is brought to you for free and open access by Journal of Marine Science and Technology. It has been accepted for inclusion in Journal of Marine Science and Technology by an authorized editor of Journal of Marine Science and Technology. 


\section{On a Computational Algorithm to the HJE in Nnlinear $\mathrm{H} \infty$ Control}

Acknowledgements

The authors are grateful to the National Science Council, Taiwan, R.O.C., for partial financial support under Contract. No. NSC-89-2213-E-019-013. 


\title{
ON A COMPUTATIONAL ALGORITHM TO THE HJE IN NONLINEAR $H_{\infty}$ CONTROL
}

\author{
Shr-Shiung Hu*, Pao-Hwa Yang** and Jeng-Yih Juang***
}

Keywords: nonlinear $H_{\infty}$ control, Hamilton-Jacobi equation, and robust control.

\begin{abstract}
The Hamilton-Jacobi equation (HJE) plays an essential role in both classical mechanics and nonlinear $H_{\infty}$ control theory. In this paper, we propose a detailed successive algorithm for finding an approximate solution of the HJE by solving linear equations. A scalar example is given to compare the computational procedures and the accuracy for the proposed approach with some other methods. In addition, the nonlinear $H_{\infty}$ controller design for the inverted pendulum is also included to show the superiority of the nonlinear $H_{\infty}$ controller, as compared with the linear $H_{\infty}$ controller, in the aspect of the robust performance/stability.
\end{abstract}

\section{INTRODUCTION}

As the linear $H_{\infty}$ control techniques [2, 4] have been developed for several years, recently the much more complicated nonlinear $H_{\infty}$ control has drawn attention to many investigators $[1,10,13,14]$ and has been solved based on the concept of energy dissipation. Ball, Helton, and Walker (BHW) [1] have successfully derived the nonlinear $H_{\infty}$ controller formulas involving two Hamilton-Jacobi inequalities (HJIs) or equations (HJEs), in which the HJEs coincidentally have the similar forms as the ones in the classical mechanics due to they both can be derived from the concept of energy. In order to obtain a nonlinear $H_{\infty}$ controller from BHW's controller formulas, one needs to solve the HJEs. Up to date, there is no computational algorithm for the exact explicit solution of HJE; however, an approximate solution can be obtained by using the successive computational methods $[3,9,11,13,15]$. Specifically, Lukes

Paper Received April 15, 2001. Author for Correspondence: Shr-Shiung Hu. *Engineering Officer, The 202nd Arsenal, C.S.F., P. O. Box 90582-22, Nankang, Taipei 115, Taiwan, R.O.C.

**Assistant Professor, Department of Mechanical Engineering, Hsiuping Institute of Technology, Dah-Li City, Taichung County 412, Taiwan, R.O.C.

***Professor, Department of Merchant Marine, National Taiwan Ocean University, Keelung 202, Taiwan, R.O.C.
[11] derived a successive algorithm for finding an approximate solution of the HJE by the power series method. Glad [3] and van der Schaft [13] simplified Lukes approach for the nonlinear input-affine system. However, their works were presented only in conceptually, not showing the detailed procedures. Wise and Sedwick (WS) [15] presented a successive approximation approach in which some integral expressions are built and used to find an approximate solution of the HJE successively. For Huang and Lin's approach [9], a computational algorithm is provided, but the approach is too complicated to employ.

In this paper, we present a successive algorithm based on $[3,13]$ in a thorough manner to show how to find an approximate solution of the HJE easily and efficiently. The proposed successive algorithm assumes that the solution of the HJE and the state equations are in the form of power series. After plugging all the information to the original HJE, one will find the linear part from the algebraic Riccati equation (ARE) is vanished and hence the higher order terms are left to form a new equation. There are two approaches to obtain an approximate solution of the HJE from the equation. One is to compare the coefficients of both sides of the equation, forming a set of linear equations, and solve them to construct an approximate solution of the HJE. The other approach uses integration instead of comparing the said coefficients. Note that the approximate solutions obtained from both approaches are identical. A higher order approximate solution can be found successively by using the above approaches if the higher accuracy is required. A scalar example will be given to demonstrate the procedure of finding an approximate solution of the HJE by the linear equation, the integration approach, and the WS [15] approach. Finally, the nonlinear $H_{\infty}$ controller design for the inverted pendulum is also included to demonstrate the design procedures and how to solve the HJE by the proposed algorithm. Simulations of the pendulum responses are also enclosed to show the superiority of the nonlinear $H_{\infty}$ controllers, as compared with its linear counterpart. 


\section{PRELIMINARIES}

\section{Nonlinear $\boldsymbol{H}_{\infty}$ Control Problem}

Consider the following nonlinear input-affine generalized plant $G$ :

$$
G:\left\{\begin{array}{l}
\dot{x}=f(x)+g_{1}(x) w+g_{2}(x) u \\
z=h_{1}(x)+D_{12}(x) u \\
y=h_{2}(x)+D_{21}(x) w
\end{array}\right.
$$

where $x \in \mathbf{R}^{n}$ is the state of the system, $z \in \mathbf{R}^{p 1}$ is the controlled output, $w \in \mathbf{R}^{m 1}$ is the exogenous input including all commands and disturbances, $u \in \mathbf{R}^{m 2}$ represents the control input, and $y \in \mathbf{R}^{p 2}$ is the measured output $[1,8,10]$. The problem is to find a controller

$$
K:\left\{\begin{array}{l}
\xi=A_{K}(\xi)+B_{K}(\xi) y \\
u=C_{K}(\xi)
\end{array}\right.
$$

so that the closed-loop system is stable and $\gamma$-dissipative $[5,8]$ or, equivalently, the $L_{2}$-gain [13] of the system is less than or equal to $\gamma$, which is a prescribed positive number.

\section{Nonlinear $\boldsymbol{H}_{\infty}$ Controller Formulas}

The nonlinear $H_{\infty}$ controller formulas in [1] are summarized in the following theorem.

Theorem 1 Consider the nonlinear generalized plant defined in (1). If there exists a controller $K$ of the form (2) such that the closed-loop system is stable and $\gamma$ dissipative, then we have the following:

(i) There exist $X(x)$ and $Y_{I}(x)$ such that the following HamiltonJacobi inequalities:

$$
\begin{aligned}
H J X(x): & =2 X^{T}(x) H_{A}(x)+X^{T}(x) H_{R}(x) X(x) \\
& +H_{Q}(x) \leq 0
\end{aligned}
$$

and

$$
H J Y_{I}(x):=2 Y_{I}^{T}(x) J_{A}(x)+Y_{I}^{T}(x) J_{R}(x) Y_{H}(x)+J_{Q}(x) \leq 0
$$

are satisfied for all $x$ in the domain of interest where

$$
\begin{aligned}
& H_{A}(x)=f(x)-g_{2}(x) E_{1}^{-1}(x) D_{12}^{T}(x) h_{1}(x) \\
& H_{R}(x)=\gamma^{-2} g_{1}(x) g_{1}^{T}(x)-g_{2}(x) E_{1}^{-1}(x) g_{2}^{T}(x) \\
& H_{Q}(x)=h_{1}^{T}(x) h_{1}(x)-h_{1}^{T}(x) D_{12}(x) E_{1}^{-1}(x) D_{12}^{T}(x) h_{1}(x) \\
& J_{A}(x)=f(x)-g_{1}(x) D_{21}^{T}(x) E_{2}^{-1}(x) h_{2}(x)
\end{aligned}
$$

$$
\begin{aligned}
J_{R}(x) & =\gamma^{-2} g_{1}(x) g_{1}^{T}(x) \\
& -\gamma^{-2} g_{1}(x) D_{21}^{T}(x) E_{2}^{-1}(x) D_{21}(x) g_{1}^{T}(x) \\
J_{Q}(x) & =h_{1}^{T}(x) h_{1}(x)-\gamma^{2} h_{2}^{T}(x) E_{2}^{-1}(x) h_{2}(x) \\
E_{1}(x) & =D_{12}^{T}(x) D_{12}(x) \\
E_{2}(x) & =D_{21}(x) D_{21}^{T}(x)
\end{aligned}
$$

(ii) $Y_{I}(x)-X(x)$ is the gradient of a positive function in the neighborhood of the equilibrium point.

(iii) A nonlinear $\gamma$-dissipative $H_{\infty}$ controller can be constructed as:

$$
\begin{aligned}
A_{K}(\xi) & =f(\xi)+\gamma^{-2}\left[g_{1}(\xi)-B_{K}(\xi) D_{21}(\xi)\right] g_{1}^{T}(\xi) X(\xi) \\
& +g_{2}(\xi) C_{K}(\xi)-B_{K}(\xi) h_{2}(\xi) \\
C_{K}(\xi) & =-E_{1}^{-1}(\xi)\left[g_{2}^{T}(\xi) X(\xi)+D_{12}^{T} h_{1}(\xi)\right]
\end{aligned}
$$

where $B_{K}(x)$ satisfies the following equation:

$$
\begin{aligned}
& {\left[Y_{I}(\xi)-X(\xi)\right]^{T} B_{K}(\xi)=\left[\gamma^{2} h_{2}^{T}(\xi)\right.} \\
& \left.\left.\quad+Y_{I}^{T}(\xi) g_{1}(\xi) D_{21}^{T}(\xi)\right)\right] E_{2}^{-1}\left(\xi_{1}\right)
\end{aligned}
$$

\section{Linearized Model and ARIs}

In order to construct a nonlinear $H_{\infty}$ controller from (5), one needs to solve the HJI or HJE. There is no exact closed-form solution available for the HJE; however, some successive approximation approaches $[3,9,11,13,15]$ can be employed to solve the HJE. The solution is in the form of power series in which the first term is constructed based on the corresponding ARE. Assume the equilibrium point is at $x=\mathbf{0}$, the linearized model of the nonlinear generalized plant described in (1) can be obtained as follows:

$$
G(s)_{\text {linear }}:\left\{\begin{array}{l}
\dot{x}=A x+B_{1} w+B_{2} u \\
z=C_{1} x+D_{12} u \\
y=C_{2} x+D_{21} w
\end{array}\right.
$$

To use a successive approximation algorithm for the solution of the HJIs, the first step is to solve their ARIs, i.e., to find $X>0$ and $Y_{I}>0$ so that the following three inequalities are satisfied.

$$
\begin{aligned}
\operatorname{Ric} X: & =\left(A-B_{2} E_{1}^{-1} D_{12}^{T} C_{1}\right)^{T} X+X\left(A-B_{2} E_{1}^{-1} D_{12}^{T} C_{1}\right) \\
& +X\left(\gamma^{-2} B_{1} B_{1}^{T}-B_{2} E_{1}^{-1} B_{2}^{T}\right) X \\
& +C_{1}^{T}\left(I-D_{12} E_{1}^{-1} D_{12}^{T}\right) C_{1} \leq 0
\end{aligned}
$$




$$
\begin{aligned}
\operatorname{RicY}_{I}: & =\left(A-B_{1} D_{21}^{T} E_{2}^{-1} C_{2}\right)^{T} Y_{I}+Y_{I}\left(A-B_{1} D_{21}^{T} E_{2}^{-1} C_{2}\right) \\
& +Y_{I} \gamma^{-2} B_{1}\left(I-D_{21}^{T} E_{2}^{-1} D_{21}\right) B_{1}^{T} Y_{I} \\
& +\left(C_{1}^{T} C_{1}-\gamma^{2} C_{2}^{T} E_{2}^{-1} C_{2}\right) \leq 0 \\
Z:= & Y_{I}-X>0
\end{aligned}
$$

where

$$
\begin{aligned}
& E_{1}=E_{1}(0)=D_{12}^{T}(0) D_{12}(0) \\
& E_{2}=E_{2}(0)=D_{21}(0) D_{21}^{T}(0)
\end{aligned}
$$

\section{HJE Approximate Solution by WS Method}

The successive approximate solution method proposed by Wise and Sedwick (WS) [15] will be briefly reviewed. Assume the nonlinearity only occurs in the system function $f(x)$ defined in (1). Define

$$
\begin{aligned}
& A_{s}=A-B_{2} E_{1}^{-1} D_{12}^{T} C_{1} \\
& R_{s}=\gamma^{-2} B_{1} B_{1}^{T}-B_{2} E_{1}^{-1} B_{2}^{T} \\
& Q_{s}=C_{1}^{T}\left(I-D_{12} E_{1}^{-1} D_{12}^{T}\right) C_{1} \\
& F_{c}=A_{s}+R_{s} X \\
& H_{A}(x)=A_{s} x+f_{h}(x)
\end{aligned}
$$

where $X \geq 0$ is the solution of the ARE (7a) and $f_{h}(x)=$ $O\left(x^{2}\right)$. The HJE (3a) can be rewritten as

$$
V_{x}(x) H_{A}(x)+\frac{1}{4} V_{x}(x) R_{s}(x) V_{x}^{T}(x)+x Q_{s} x^{T}=0
$$

where

$$
V_{x}=\frac{\partial V}{\partial x}=2 X^{T}(x)
$$

and the corresponding ARE (7a) becomes

$$
A_{s}^{T} X+X A_{s}+X R_{s} X+Q_{s}=0
$$

The WS successive approach is summarized as follows. Define

$$
\begin{aligned}
& J(t)=\int_{0}^{t} e^{F_{c} \xi}\left(\frac{1}{2} R_{s}\right) e^{F_{c}^{T} \xi} d \xi \\
& q(z, p)=-2 \frac{\partial^{T} f_{h}(z)}{\partial z} X z-\frac{\partial^{T} f_{h}(z)}{\partial z} p-2 X f_{h}(z) \\
& z(t)=e^{F_{c} t} z(0)+J(t) p(t)+e^{F_{c} t} \int_{0}^{t} e^{-F_{c} \tau} f_{h}(z(\tau)) \\
& \quad-J(\tau) q(z(\tau), p(\tau))] d \tau
\end{aligned}
$$

$$
p(t)=-e^{-F_{c}^{T} t} \int_{0}^{t} e^{F_{c}^{T} \tau} q(z(\tau), p(z)) d \tau
$$

The successive computing procedure starting by setting the zero-th approximation as

$$
z_{(0)}(t)=e^{F_{c} t} z \text { and } p_{(0)}(t)=0
$$

then plugging (12) into (11) to obtain the first approximation. Repeat to compute (11) by using the latest $z(t)$ and $p(t)$ until generate the desired degree of approximation. The approximate solution of $V_{x}(x)$ can be computed by

$$
V_{x}(x)=2 x^{T} X+\left.p(t)\right|_{t=0}
$$

\section{A DETAILED COMPUTATIONAL ALGORITHM FOR SOLVING THE HJE}

In order to obtain a nonlinear $H_{\infty}$ controller from Theorem 1, one needs to solve the HJEs. In [3, 13], the method of solving the HJE was only presented conceptually without showing detailed procedures. In this section, we will present a modified successive algorithm in a thorough manner to construct an approximate solution for the HJE which is the main contribution of the paper. The HJE (3a) can be rewritten as:

$$
V_{x}(x) H_{A}(x)+\frac{1}{4} V_{x}(x) H_{R}(x) V_{x}^{T}(x)+H_{Q}(x)=0
$$

where $V_{x}(x)$ is defined in (9b). Define $A_{s}, R_{s}, Q_{s}, F_{c}$, and $H_{A}(x)$ as the same in (8). Let, $R_{h}(x)=O(x)$ and $Q_{h}(x)=$ $O\left(x^{3}\right)$ be the high-order terms that satisfy the following,

$$
\begin{aligned}
& \frac{1}{4} H_{R}(x)=\frac{1}{4} R_{s}+R_{h}(x) \\
& H_{Q}(x)=x^{T} Q_{s} x+Q_{h}(x)
\end{aligned}
$$

Recall that $(\bullet)^{(k)}$ denotes the k-th order term , $(\bullet)^{[k]}$ stands for the sum of the accumulated terms up to the $k$ th order term, and $X$ is the positive semi-definite stabilizing solution of the ARE (10). Now, the $k$-th order approximate solution of (14) can be written as

$$
V^{[k]}(x)=\sum_{m=2}^{k} V^{(m)}(x)=x^{T} X x+\sum_{m=3}^{k} V^{(m)}(x)
$$

and its derivative will be

$$
\frac{\partial V^{[k]}}{\partial x}=2 x^{T} X+\sum_{m=3}^{k} \frac{\partial V^{(m)}}{\partial x}
$$

Note that the order of $\frac{\partial V^{(m)}}{\partial x}$ is $m-1$. Using (8e), (15a), and (15b), we approximate (14) to the following:

$$
\frac{\partial V^{[K]}}{\partial x}\left[A_{s} x+f_{h}(x)\right]+\frac{1}{4} \frac{\partial V^{[K]}}{\partial x}\left[R_{s}+4 R_{h}(x)\right] \frac{\partial^{T} V^{[K]}}{\partial x}
$$




$$
+x^{T} Q_{s} x+Q_{h}(x)
$$

$=\frac{\partial V^{[K]}}{\partial x} A_{s} x+\frac{\partial V^{[K]}}{\partial x} f_{h}(x)+\frac{1}{4} \frac{\partial V^{[K]}}{\partial x} R_{s} \frac{\partial^{T} V^{[K]}}{\partial x}$

$+\frac{\partial V^{[K]}}{\partial x} R_{h}(x) \frac{\partial^{T} V^{[K]}}{\partial x}+x^{T} Q_{s} x+Q_{h}(x)=0$

From (16), equation (17) can be expressed as

$$
\begin{aligned}
& \underline{2 x^{T} X A_{s} x}+\sum_{m=3}^{k} \frac{\partial V^{(m)}}{\partial x} A_{s} x+\frac{\partial V^{[K]}}{\partial x} f_{h}(x)+\underline{x^{T} X R_{s} X x} \\
& +\sum_{m=3}^{k} \frac{\partial V^{(m)}}{\partial x} \frac{1}{4} R_{s} \frac{\partial V^{(m)}}{\partial x}+\sum_{m=3}^{k} \frac{\partial V^{(m)}}{\partial x} R_{s} X x \\
& +\frac{\partial V^{[K]}}{\partial x} R_{h}(x) \frac{\partial^{T} V^{[K]}}{\partial x}+\underline{x^{T} Q_{s}} x+Q_{h}(x)=0
\end{aligned}
$$

Collecting all the underlined terms in (18) and from (10) we have

$$
\begin{aligned}
& 2 x^{T} X A_{s} x+x^{T} X R_{s} X x+x^{T} Q_{s} x \\
& \quad=x^{T}\left(A^{T} x+X A+X R_{S} X+Q\right) x=0
\end{aligned}
$$

From (19) and (8d), the equation (18) can be rearranged as

$$
\begin{aligned}
& \sum_{m=3}^{k} \frac{\partial V^{(m)}}{\partial x} F_{c} x+\frac{\partial V^{[K]}}{\partial x} f_{h}+\sum_{m=3}^{k-1} \frac{\partial V^{(m)}}{\partial x} \frac{1}{4} R_{s} \sum_{m=3}^{k-1} \frac{\partial^{T} V^{(m)}}{\partial x} \\
& +\frac{\partial V^{[k]}}{\partial x} R_{h} \frac{\partial^{T} V^{[K]}}{\partial x}+Q_{h}=0
\end{aligned}
$$

In order to find $\frac{\partial V^{(k)}}{\partial x}$ successively, one needs to put the first term of (20) in the left hand side of a new equation and all other terms in (20) using the solutions from the previous iteration to form the right hand side of the new equation, i.e.,

$$
\begin{aligned}
& -\sum_{m=3}^{k} \frac{\partial V^{(m)}}{\partial x} F_{c} x=\frac{\partial V^{[k-1]}}{\partial x} f_{h}+\sum_{m=3}^{k-1} \frac{\partial V^{(m)}}{\partial x} \frac{1}{4} R_{s} \sum_{m=3}^{k-1} \frac{\partial^{T} V^{(m)}}{\partial x} \\
& +\frac{\partial V^{[k-1]}}{\partial x} R_{h} \frac{\partial^{T} V^{[k-1]}}{\partial x}+Q_{h}
\end{aligned}
$$

Note that in (21) all the terms whose order is less than $k$ will be vanished due to the cancellation of the previous successive procedures and all terms whose order are grater than $k$ are ignored during the $k$-th order iteration and hence $(21)$ becomes a pure $k$-th order equation and the approximate solutions of the HJE (3a) can be computed successively based on the following equation:

$$
\begin{aligned}
& -\frac{\partial V^{(k)}}{\partial x} F_{c} x=\sum_{m=2}^{k-1} \frac{\partial V^{(m)}}{\partial x} f_{h}^{(k-m+1)} \\
& +\sum_{m=3}^{k-1} \frac{\partial V^{(k-m+2)}}{\partial x} \frac{1}{4} R_{s} \frac{\partial^{T} V^{(m)}}{\partial x}
\end{aligned}
$$

$$
+\sum_{n=1}^{k-2 k-n} \sum_{m=2} \frac{\partial V^{(k-n-m+2)}}{\partial x} R_{h}^{(n)} \frac{\partial^{T} V^{(m)}}{\partial x}+Q_{h}^{(k)}:=H_{m}^{(k)}(x)
$$

where $k \geq 3$ is an integer. By comparing the coefficients on both sides of (22), a set of linear equations are established and employed to solve $V^{(k)}$. Then, based on (9b), an approximate solution of the HJE in (3a), $X(x)$, is constructed as follows,

$$
X^{[k-1]}(x)=\frac{1}{2} \frac{\partial^{T} V^{[k]}}{\partial x}=X^{[k-2]}(x)+\frac{\partial^{T} V^{(k)}}{\partial x}
$$

\section{Computational Procedure}

(i) The first-order approximate solution:

$$
X^{[1]}(x)=\frac{1}{2} \frac{\partial^{T} V^{(2)}}{\partial x}=X x
$$

where $X$ is defined in (10).

\section{(ii) The second-order approximate solution:}

Assume the number of possible third-order terms of $x$ is $n_{3}$. Let $V^{(3)}(x)$ be the linear combination of these $n_{3}$ terms. For example, if $x=\left[\begin{array}{lll}x_{1} & x_{2} & x_{3}\end{array}\right]^{T}$, then

$$
\begin{aligned}
V^{(3)}(x) & =c_{1} x_{1}^{3}+c_{2} x_{2}^{3}+c_{3} x_{3}^{3}+c_{4} x_{1}^{2} x_{2}+c_{5} x_{1}^{2} x_{3}+c_{6} x_{1} x_{2}^{2} \\
& +c_{7} x_{1} x_{3}^{2}+c_{8} x_{1} x_{2} x_{3}+c_{9} x_{2}^{2} x_{3}+c_{10} x_{2} x_{3}^{2}
\end{aligned}
$$

and $n_{3}=10$. Equation (22) now becomes

$$
-\frac{\partial V^{(3)}(x)}{\partial x} F_{c} x=2 x^{T} X f_{h}^{(2)}(x)+4 x^{T} X R_{h}^{(1)}(x) X x+Q_{h}^{(3)}(x)
$$

Note that both sides of (26) consist of only thirdorder terms. By comparing the coefficients for both sides of (26), a set of $n_{3}$ linear equations are established to give the solution $V^{(3)}(x)$. Then the second-order approximate solution from (23) is

$$
X^{[2]}(x)=X x+\frac{1}{2} \frac{\partial^{T} V^{(3)}}{\partial x}
$$

(iii) The third-order approximate solution:

As before, assume $V^{(4)}(x)$ has $n_{4}$ forth-order terms of $x$. Equation (22) now is

$$
\begin{aligned}
-\frac{\partial V^{(4)}}{\partial x} F_{c} x & =2 x^{T} X f_{h}^{(3)}+\frac{\partial V^{(3)}}{\partial x} f_{h}^{(2)} \\
& +\frac{\partial V^{(3)}}{\partial x} \frac{1}{4}\left(\gamma^{-2} B_{1} B_{1}^{T}-B_{2} B_{2}^{T}\right) \frac{\partial^{T} V^{(3)}}{\partial x} \\
& +4 \frac{\partial V^{(3)}}{\partial x} R_{h}^{(1)} X x+4 x^{T} X R_{h}^{(2)} X x+Q_{h}^{(4)}
\end{aligned}
$$


Note that the only nonlinear terms involved in (28) are $f_{h}^{(2)}, f_{h}^{(3)}, R_{h}^{(1)}, R_{h}^{(2)}$, and $Q_{h}^{(4)}$; hence $V^{(4)}(x)$ can be found as a solution of the $n_{4}$ linear equations obtained from comparing the coefficients of (28). The third-order approximate solution $X^{[3]}(x)$ is

$$
X^{[3]}(x)=X^{[2]}(x)+\frac{1}{2} \frac{\partial^{T} V^{(4)}}{\partial x}
$$

The successive computation procedure can continue to produce higher order approximations if higher accuracy is required.

\section{Remark 1}

(i) From the above detailed procedure of finding an approximate solution of the HJE, we know that the number of terms, i.e. $n_{k}$, involved in $V^{(k)}(x)$ during the $k$-th order iteration is essential for applying the proposed successive algorithm. Actually the $n_{k}$ can be simply represented [12] by $\left(\begin{array}{c}n+k-1 \\ n-1\end{array}\right)$ where $\mathrm{n}$ is
the size of the system.

(ii) One question arises on how to make sure that the approximate solution computed is correct. Here we provide a method to check the solution. Once the approximate solution $X^{[k-2]}(x)(k \geq 3)$ is obtained and plug it in (3a), you will see that the terms $k$ whose order is less than $\mathrm{k}$ is vanished.

(iii) Another method for finding $V^{(k)}(x)$ is to use the integration method $[11,13]$. Due to $F_{c}$ being a stable matrix, $V^{(k)}(x)$ can be calculated from (22) by

$$
V^{(k)}(x)=\int_{0}^{\infty} H_{m}^{(k)}\left(e^{F c^{t}} x\right) d t
$$

where $H_{m}^{(k)}$ is defined in (22). Note that $V^{(k)}(x)$ should be identical for both the proposed linear equations method and the integration method (30).

\section{A SCALAR EXAMPLE}

In this section, a scalar example which was originally presented by WS [15] is given to demonstrate finding an approximate solution for the HJE by using the three approaches mentioned in the previous sections, i.e., the WS method, the proposed linear equations method, and the integration method. Consider the following HJE with the form of (14) as

$$
V_{x}\left[-7 x+f_{h}(x)\right]-\frac{1}{4} V_{x}^{2}+15 x^{2}=0
$$

whose exact solution can be easily computed as

$$
V_{x}=2 x\left(-7+\frac{f_{h}(x)}{x}+\sqrt{\left(-7+\frac{f_{h}(x)}{x}\right)^{2}+15}\right)
$$

$=2 x+\frac{1}{4} f_{h}+\frac{15}{8^{3}} \frac{f_{h}^{2}}{x}+\frac{105}{8^{5}} \frac{f_{h}^{3}}{x^{2}}+\cdots$

Assume $f_{h}(x)=a x^{3}$ where $a$ is a constant, then (32b) becomes

$$
V_{x}=2 x+\frac{1}{4} a x^{3}+\frac{15}{8^{3}} a^{2} x^{5}+\frac{105}{8^{5}} a^{3} x^{7}+\cdots
$$

The ARE in (10) for the linearized model is:

$$
X^{2}+14 X-15=0
$$

whose positive solution is $X=1$. From (8) and (10), it is easy to see that $R_{s}=-1, F_{c}=-8, R_{h}(x)=0$, and $Q_{h}(x)$ $=0$.

(M1) The WS method

From (11), we can get

$$
\begin{aligned}
& J(t)=\frac{1}{32}\left(e^{16 t}-1\right) \\
& a(x, p)=-8 a x^{3}-3 a x^{2} p \\
& (t)=e^{-8 t} z(0)+\frac{1}{32}\left(e^{-16 t}-1\right) p(t)+a e^{-8 t} \int_{0}^{t} e^{8 \tau}\left[z^{3}\right. \\
& \left.+\frac{1}{32}\left(e^{-16 \tau}-1\right)\left(8 z^{3}+3 z^{2} p\right)\right] d \tau \\
& p(t)=a e^{8 t} \int_{0}^{t} e^{-8 \tau}\left[8 z^{3}+3 z^{2} p\right] d \tau
\end{aligned}
$$

in which the zeroth approximation is assumed as

$$
z_{(0)}(t)=e^{-8 t} x \text { and } p_{(0)}(t)=0
$$

Plugging (36) into $(35 \mathrm{c}, \mathrm{d})$ results in the first approximation as

$$
z_{(1)}(t)=e^{-8 t} x-a x^{3}\left(-\frac{7}{128} e^{-8 t}+\frac{3}{64} e^{-24 t}-\frac{1}{128} e_{(37 a)}^{-40 t}\right)
$$

$p_{(1)}=\frac{1}{4} a e^{-24 t} x^{3}$

Repeat the same procedure by plugging (37) into $(35 \mathrm{c}, \mathrm{d})$ again, we have

$p_{(2)}(t=0)=a\left[\frac{1}{4} x^{3}+\frac{31}{2^{10}} a x^{5}+\frac{289}{2^{15} \cdot 10} a^{2} x^{7}+\cdots\right]$

By using (13), $V_{x}(x)$ can be obtained as:

$$
\left.V_{x}(x)=2 x+\frac{1}{4} a x^{3}+\frac{31}{2^{10}} a^{2} x^{5}+\frac{289}{2^{15} \cdot 10} a^{3} x^{7}+\cdots\right]
$$

(M2) The linear equations method

Next, we consider the method based on the con- 
struction of the linear equations from comparing the coefficients of both sides of (22). Starting from $k=3$, it is easy to see that $V^{(3)}(x)=0$ because of $f_{h}^{(2)}=R_{h}^{(1)}=Q_{h}^{(3)}$ $=0$. Note that in this example, $V^{(2 m+1)}(x)=0$ for all $m \in$ $N$. For $k=4$, we have $V^{(4)}(x)=b_{4} x^{4}$ where $b_{4}$ is a constant to be determined. Equation (22) can be rewritten as

$$
-\frac{\partial V^{(4)}}{\partial x} F_{c} x=2 x X f_{h}^{(3)}
$$

Plugging all given numbers into (40a) results in

$$
-4 b_{4} x^{3}(-8) x=2 x(1) a x^{3}=2 a x^{4}:=H_{m}^{(4)}(x)
$$

Comparing the coefficient of $x^{4}$ for both sides, we can construct one linear equation as $32 b_{4}=2 a$ which gives $b_{4}=\frac{1}{16 a}$ and hence $V_{x}^{(4)}=\frac{1}{4} a x^{3}$. For $k=6$, by assuming $V^{(6)}(x)=b_{6} x^{6}$, Equation (18) can be rewritten as

$$
\begin{aligned}
& -\frac{\partial V^{(6)}}{\partial x} F_{c} x=\frac{\partial V^{(4)}}{\partial x} f_{h}^{(3)}+\frac{1}{4}\left(\frac{\partial V^{(4)}}{\partial x}\right)^{2} R_{s} \\
& -6 b_{6} x^{5}(-8) x=\frac{15}{64} a^{2} x^{6}:=H_{m}^{(6)}(x)
\end{aligned}
$$

which gives $b_{6}=\frac{15}{6 \times 8^{3}} a^{2}$. The same procedure can be repeated to get $V^{(8)}(x)=b_{8} x^{4}$ with $b_{8}=\frac{105}{8^{6}}$. Then we have the following approximate solution 8

$$
V_{x}^{[8]}(x)=2 x+\frac{1}{4} a x^{3}+\frac{15}{8^{3}} a^{2} x^{5}+\frac{105}{8^{5}} x^{7}
$$

(M3) The integration method

The method is the same as M2 with the exception that the integration method is applied to find $V_{x}^{(k)}(x)$. As in M2, we have $V^{(2 m+1)}=0$ for $m \in N . V^{(4)}(x)$ can be calculated by (30) and (40b) as

$$
V^{(4)}(x)=2 a \int_{0}^{\infty}\left(e^{-8 t} x\right)^{4} d t=\frac{1}{16} a x^{4}
$$

$V^{(6)}(x)$ can be calculated by (30) and (41b) as

$$
V^{(6)}(x)=\frac{15}{64} a^{2} \int_{0}^{\infty}\left(e^{-8 t} x\right)^{6} d t=\frac{15}{6 \times 8^{3}} a^{2} x^{6}
$$

\section{Remark 2}

(i) The solution from M3 is the same as the one from M2 because $V^{(k)}(x)$ is the unique solution by comparing the coefficient from (18). Actually, the solution (42) from M2 to the exact solution defined in (33) with the terms higher than seventh-order truncated. On the other hand, the solution (39) from M1 is slightly different from the truncated exact solution. In Fig. 1 , the linear solution, the third-order approximate solution for both approaches, the seventh-order ap- proximate solutions (39) by M1 and (42) by M2 are compared with the exact solution (32b). From Fig. 1 , we can see that higher order solution is closer to the exact solution. In this particular example, the solution from M2 is more accurate than the one from M1.

(ii) Both M1 and M3 involve integration computations which may require impractical computing time when the matrix size of $F_{c}$ is large. Method M2 requires only solving linear equations which is less completed than integration.

\section{NONLINEAR $H_{\infty}$ CONTROLLER DESIGN FOR INVERTED PENDULUM}

The nonlinear model of the Inverted pendulum [5] is represented as:

$$
\begin{aligned}
& {\left[\begin{array}{l}
r \\
\dot{r} \\
\theta \\
\dot{\theta}
\end{array}\right]:=\left[\begin{array}{l}
\dot{x}_{1} \\
\dot{x}_{2} \\
\dot{x}_{3} \\
\dot{x}_{4}
\end{array}\right]=\left[\begin{array}{c}
x_{2} \\
0 \\
x_{4} \\
24.1314 x_{3}
\end{array}\right]+\left[\begin{array}{c}
0 \\
0 \\
0 \\
-4.0219 x_{3}^{3}
\end{array}\right]} \\
& +\left[\begin{array}{c}
0 \\
1 \\
0 \\
-2.4606
\end{array}\right] u+\left[\begin{array}{c}
0 \\
0 \\
0 \\
1.2303 x_{3}^{2}
\end{array}\right] u \\
& :=f(x)+g_{2}(x) u:=A x+f_{h}(x)+B_{2} u+g_{2 h}(x) u
\end{aligned}
$$

where $r$ is the cart displacement and $\theta$ is the pendulum angle. The measured outputs are $r$ and $\theta$, so the output equations can be represented by

$$
y=\left[\begin{array}{l}
r \\
\theta
\end{array}\right]=\left[\begin{array}{l}
x_{1} \\
x_{3}
\end{array}\right]=\left[\begin{array}{llll}
1 & 0 & 0 & 0 \\
0 & 0 & 1 & 0
\end{array}\right] x:=C_{2} x
$$

The objective is to design a (nonlinear $H_{\infty}$ ) con-

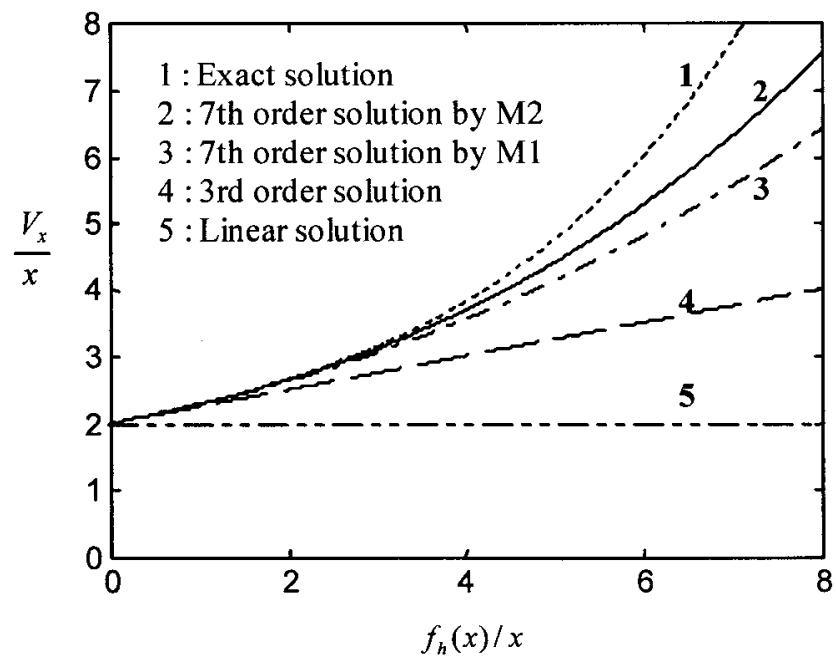

Fig. 1. Comparison of different approaches with the exact solution. 
troller to drive the cart motor so that the cart can move back and forth maintaining the stick at a strictly vertical position (keeping $\theta=0$ ). From the nonlinear $H_{\infty}$ control problem formulation [6], we assume that a disturbance $d$ is injected into the system via the state equation and the measurement is contaminated by the noise $n$. The weighted state vector, $z_{1}$, represents the disturbance response of interest to be minimized. The weighted control input, $z_{2}$, is employed to add control input constraint into the problem formulation. $W_{n}, W_{x}, W_{u}$, and $W_{n}$ are appropriate constant weighting matrices. Let $w=\left[\begin{array}{ll}d & n\end{array}\right]^{T}$ and $z=\left[\begin{array}{ll}z_{1} & z_{2}\end{array}\right]^{T}$, then the nonlinear generalized plant for the inverted pendulum control problem formulation is constructed as follows,

$$
\begin{aligned}
\dot{x} & =f(x)+g_{1}(x) w+g_{2}(x) u \\
& =f(x)+\left[\begin{array}{ll}
W_{d} & 0
\end{array}\right] w+g_{2}(x) \mathrm{u}: \\
& =A x+f_{h}(\mathrm{x})+B_{1} w+B_{2} u+g_{2 h}(x) u \\
z & =\left[\begin{array}{l}
z_{1} \\
z_{2}
\end{array}\right]=h_{1}(x)+D_{12}(x) u=\left[\begin{array}{c}
W_{x} \\
0
\end{array}\right] x+\left[\begin{array}{c}
0 \\
W_{u}
\end{array}\right] u \\
y & =h_{2}(x)+D_{21}(x) w=C_{2} x+\left[\begin{array}{ll}
0 & W_{n}
\end{array}\right] w
\end{aligned}
$$

The weighting matrices $W_{x}, W_{d}, W_{n}$, and $W_{u}$ are chosen as:

$$
W_{x}=\left[\begin{array}{cccc}
1 & 0 & 0 & 0 \\
0 & 10^{-6} & 0 & 0 \\
0 & 0 & 1 & 0 \\
0 & 0 & 0 & 10^{-6}
\end{array}\right], \quad W_{d}=I_{4}, W_{n}=I_{2},
$$

and $W_{u}=1$

The first step is to consider the linearized model of (46). The optimal $H_{\infty}$ norm of the linear closed-loop system is computed [2] as $\gamma_{\text {opt }}=52.42$. Choosing $\gamma=55$ $>\gamma_{o p t}$, one can find the solution for the ARE in (10) as

$$
X=\left[\begin{array}{cccc}
1.8258 & 1.6660 & 5.3303 & 1.0859 \\
1.6660 & 2.4959 & 8.6347 & 1.7594 \\
5.3303 & 8.6347 & 69.6382 & 14.1508 \\
1.0859 & 1.7594 & 14.1508 & 2.8797
\end{array}\right]
$$

The first-order approximate solution of the HJE from (24) is

$$
X^{[1]}(x)=X x
$$

where $X$ is given in (48). Due to $f_{h}^{(2)}(x)=R_{h}^{(1)}(x)=Q_{h}^{(3)}(x)$ $=0$ and from (26), we see that $V^{(3)}(x)=0$. The secondorder approximate solution is the same as the first-order one, i.e.,

$$
X^{[2]}(x)=X^{[1]}(x)=X x
$$

The construction of the third-order approximate solution is explained as follows. First, we compare the coefficients of the terms on both sides of (28) and set up 35 linear equations $\left(n_{4}=35\right.$, see Remark 1 (i)), which in turn will be solved for $V^{(4)}(x)$. Then we have the thirdorder approximate solution $X^{[3]}(x)$ according to (29),

$$
X^{[3]}(\mathrm{x})=X x+X^{(3)}(x)
$$

where

$$
\begin{aligned}
X^{(3)}(x): & =\left[\begin{array}{c}
X_{1}^{(3)} \\
X_{2}^{(3)} \\
X_{3}^{(3)} \\
X_{4}^{(3)}
\end{array}\right] \\
X_{1}^{(3)}(x)= & 0.07290 x_{1} x_{3}^{2}+0.1240 x_{1} x_{3}^{2}+2.3450 x_{3}^{3} \\
& +0.6130 x_{3}^{2} x_{4} \\
X_{2}^{(3)}(x)= & 0.1240 x_{1} x_{3}^{2}+0.2055 x_{2} x_{3}^{2}+3.9339 x_{3}^{3} \\
& +1.0069 x_{3}^{2} x_{4} \\
X_{3}^{(3)}(x)= & 0.2480 x_{1} x_{2} x_{3}+0.2055 x_{2}^{2} x_{3}+7.0348 x_{1} x_{3}^{2} \\
& +11.8017 x_{2}+98.6658 x_{3}^{3}+1.2260 x_{1} x_{3} x_{4} \\
& +2.0137 x_{2} x_{3} x_{4}+37.5422 x_{3}^{2} x_{4}+3.9021 x_{3} x_{4}^{2} \\
& +0.07290 x_{1}^{2} x_{3}+0.09527 x_{4}^{2} \\
X_{4}^{(3)}(x)= & 0.6130 x_{1} x_{3}^{2}+1.0069 x_{2} x_{3}^{2}+12.5141 x_{3}^{3} \\
& +3.9021 x_{3}^{2} x_{4}+0.2858 x_{3} x_{4}^{2}+0.04679 x_{2} x_{3} x_{4}
\end{aligned}
$$

For simplicity, the coefficients with absolute value less than 0.04 were chopped. A nonlinear $H_{\infty}$ controller can be obtained by plugging the third-order approximate solution (51) of the HJE to the controller formulas (5).

\section{Simulations}

The computer simulations for the closed-loop system will be performed. Let the initial conditions be [0 $\left.\begin{array}{lll}0 & \theta_{0} & 0\end{array}\right]^{T}$ and the exogenous input be

$$
w=A_{w}\left[\begin{array}{llllll}
1 & 1 & 1 & 1 & 1 & 1
\end{array}\right]^{T}
$$

where $A_{w}$ is a constant representing the disturbance 
amplitude. First, the simulation is performed under the condition of no disturbance. The pendulum responses with $A_{w}=0$ and small initial angle $\theta_{0}=0.2$ are plotted in Fig. 2. It shows that the pendulum angle $\theta$ converges to 0 after 3 seconds and the cart displacement $r$ only deviates a little bit before it quickly returns to its equilibrium. Simulations for the linear $H_{\infty}$ controller show that the performance of the linear controller is almost the same as that of the nonlinear controller at this point.

Next we increase the initial pendulum angular displacement to $\theta_{0}=0.5$ and let the disturbance amplitude be $A_{w}=0.15$. The $\theta$ responses for both the nonlinear $H_{\infty}$ and linear $H_{\infty}$ controllers are plotted in Fig. 3 . It is obvious that the nonlinear $H_{\infty}$ controller has better performance in keeping the stick straight. If $A_{w}$

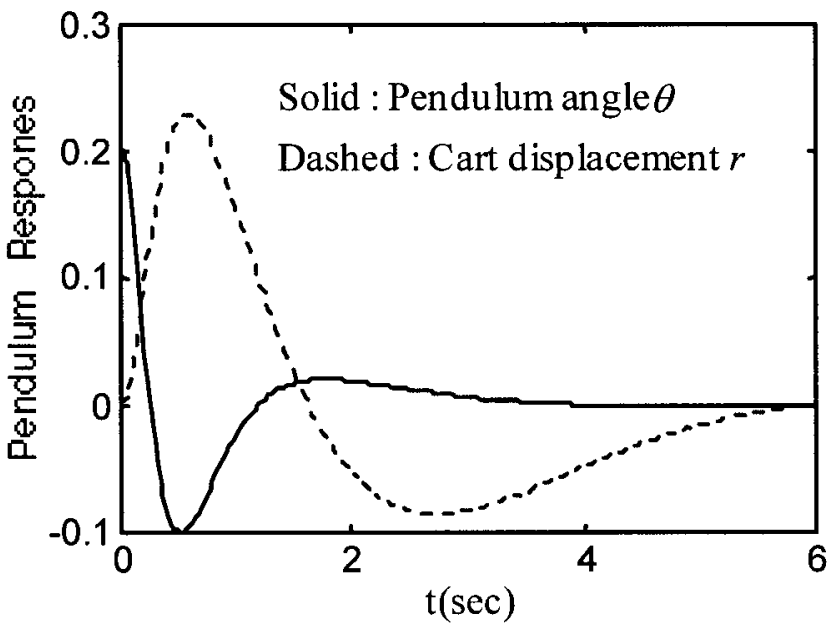

Fig. 2. Pendulum responses with the nonlinear $H_{\infty}$ controller when $\theta_{0}=$ 0.2 and $A_{w}=0$.

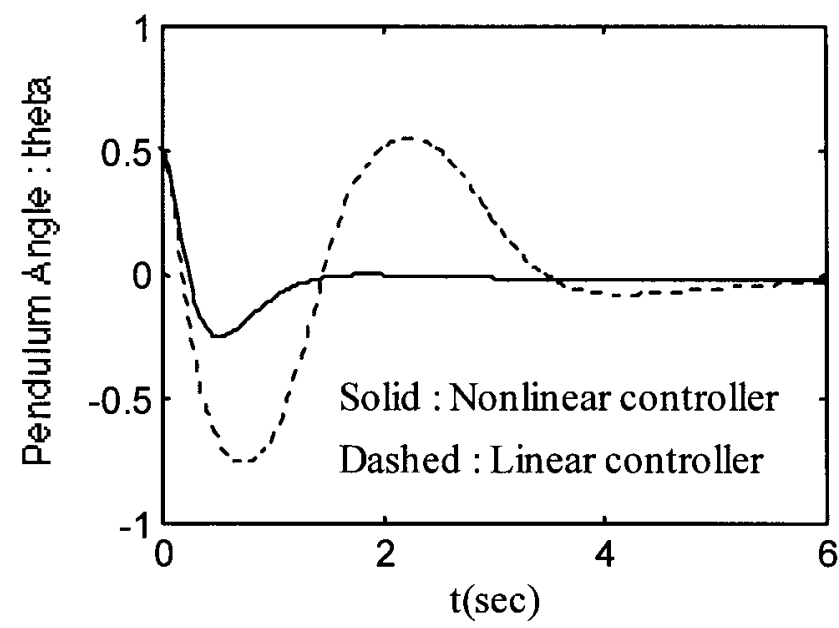

Fig. 3. Comparison of pendulum angle $\theta$ responses when $\theta_{0}=$ 0.5 and $A_{w}=0.15$ is increased even larger to 0.2 , then the $\theta$ response for the linear $H_{\infty}$ controller diverges while the response for the nonlinear $H_{\infty}$ controller is able to converge to zero after only 4 seconds.

\section{CONCLUSIONS}

In this paper, a detailed successive algorithm and computational procedure for finding an approximate solution of the HJE by solving the linear equations were presented. A scalar example was given to compare three approaches: the WS approach, the proposed linear equations method, and the integration method and it was found that the proposed successive algorithm - linear equations method (M2) is the best approach among the three methods. The proposed algorithm was also employed to find an approximate solution for the HJE leading to construct a nonlinear $H_{\infty}$ controller for the inverted pendulum. Simulations of the closed-loop pendulum responses for both nonlinear and linear controllers were performed and it was found that the nonlinear $H_{\infty}$ controller has better performance and robustness than the linear controller, which reveals the importance of the proposed algorithm of solving the HamiltonJacobi equation.

\section{ACKNOWLEDGMENTS}

The authors are grateful to the National Science Council, Taiwan, R.O.C., for partial financial support under Contract. No. NSC-89-2213-E-019-013.

\section{NOMENCLATURE}

$\begin{array}{ll}\text { ARE } & \text { algebraic Riccati equation } \\ \text { ARI } & \text { algebraic Riccati inequality } \\ \text { BHW } & \text { Ball, Helton, and Walker } \\ \text { HJE } & \text { Hamilton-Jacobi equation } \\ \text { HJI } & \text { Hamilton-Jacobi inequality } \\ O\left(x^{m}\right) & \text { the higher order terms including } x^{m} \\ \mathbf{R}^{n} & \text { n-dimensional Euclidean space } \\ \text { WS } & \text { Wise and Sedwick } \\ (\bullet)^{(k)} & \text { the } k \text {-th order term } \\ (\bullet)^{[k]} & \text { the sum of all terms up to the } k \text {-th order term }\end{array}$

\section{REFERENCES}

1. Ball, J. A., Helton, J. W., and Walker, M. L, " $H_{\infty}$ Control for Nonlinear Systems with Output Feedback," IEEE Trans. on Automatic Control, Vol. 38, pp. 545-559 (1993).

2. Doyle, J. C., Glover, K., Khargonekar, P. P., and Francis, B. A., "State Space Solutions to Standard $H_{2}$ and $H_{\infty}$ Control Problems," IEEE Trans. on Automatic Control, 
Vol. 34, pp. 831-846 (1989).

3. Glad, S.T., "Robustness of Nonlinear State Feedback - A Survey," Automatica, Vol. 23, No. 4, pp. 425-435 (1987).

4. Glover, K., and Doyle, J. C., "State-space Formula for All Stabilizing Controllers that Satisfy an $H_{\infty}$-norm Bound and Relations," Systems \& Control Letters 11, pp. 167-172 (1988).

5. Hu, S. S. and Chang, B. C., "Design of a Nonlinear $H_{\infty}$ Controller for the Inverted Pendulum System," Proceedings of the 1998 IEEE Conference on Control Applications, pp. 699-703 (1998).

6. Hu, S. S., Yang, P. H., and Chang, B. C., "Modified Nonlinear $H_{\infty}$ Controller Formulas and the $H_{\infty}$ I/O Linearization Problem", Proceedings of the 1998 IEEE Conference on Decision and Control, pp. 3500-3505 (1998).

7. Hu, S. S., Yang, P. H., and Chang, B. C., " A Successive Algorithm for Solving the Hamilton-Jacobi Equations," Proceedings of the 1999 American Control Conference, pp. 2842-2846 (1999).

8. Yang, P. H., Hu, S. S., Su, C. M., and Juang, J. Y., "On The Nonlinear $H_{\infty}$ Controller Design For A Ship CourseKeeping Problem Via Energy Dissipation Approach," Journal of the Society of Naval Architects and Marine Engineers, R.O.C., Vol. 20, No. 1, pp. 49-59 (2001).

9. Huang, J. and Lin, C. F., "Numerical Approach to Computing Nonlinear $H_{\infty}$ Control Laws," Journal of Guidance, Control, and Dynamics, Vol. 18, No. 5, pp. 989-994 (1995).

10. Isidori, A. and Astolfi, A., "Disturbance Attenuation and $H_{\infty}$-Control Via Measurement Feedback in Nonlinear Systems," IEEE Trans. on Automatic Control, Vol. 37., No. 9 (1992).

11. Lukes, D. L., "Optimal Regulation of Nonlinear Dynamical Systems," SIAM J. Optm. Control, Vol. 7, pp. 75-100 (1969).

12. Patpong, L., Sampei, M., Koga, M., and Shimizu, E., “A Numerical Computational Approach of Hamilton-JacobiIsaacs Equation in Nonlinear $H_{\infty}$ Control Problems," Proceedings of the 35th Conference on Decision and Control, pp. 3774-3779 (1996).
13. Van der Schaft, A. J., " $L_{2}$-gain Analysis of Nonlinear Systems and Nonlinear $H_{\infty}$ Control," IEEE Trans. on Automatic Control, Vol. 37, pp. 770-784 (1992).

14. Van der Schaft, A. J., $L_{2}$-Gain and Passivity Techniques in Nonlinear Control, Spriger, London (1996).

15. Wise, K. A. and Sedwick, J. L., "Successive Approximation Solution of the HJI Equation," Proceedings of the 33rd Conference on Decision and Control, pp. 13871391 (1994).

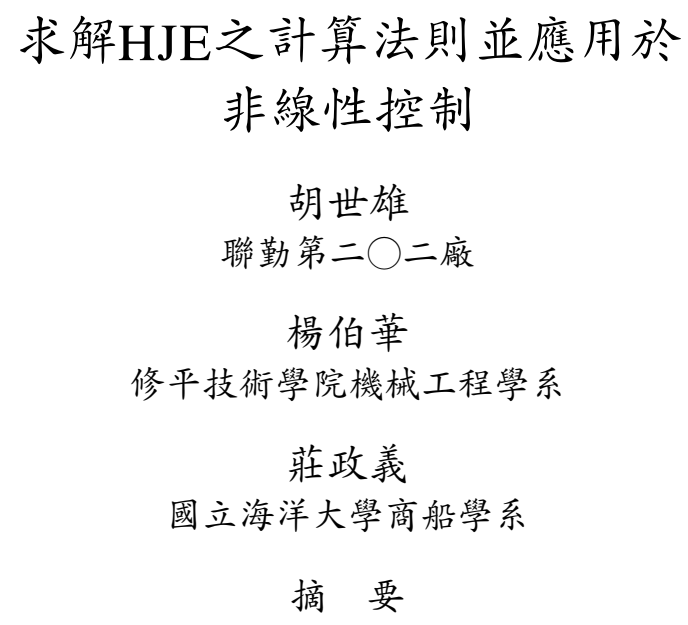

在古典力學及非線性 $H_{\infty}$ 控制理論, HamiltonJacobi方程式(HJE)均扮演著重要的角色。在本論文 中, 我們提出了一個詳細的疊代法則及計算程序, 以 解線性方程式來求得HJE之近似解。文中附上一個純 量的範例來比較所提之計算法則與其他方法之求解過 程。最後, 本論文探討一個控制的實例, 即設計倒單 擺之非線性 $H_{\infty}$ 控制器, 並驗證其與線性 $H_{\infty}$ 控制器比 較之強幃性能/稳定性的優越性, 以顯示本計算法則 之必要性。

關鍵詞：非線性 $H_{\infty}$ 控制、Hamilton-Jacobi方程式、 強健控制。 